\title{
Kernos
}

Revue internationale et pluridisciplinaire de religion grecque antique

19 | 2006

Varia

\section{Christoph RIEDWEG, Pythagoras. His Life, Teaching, and Influence}

\section{André Motte}

\section{OpenEdition}

\section{Journals}

Édition électronique

URL : https://journals.openedition.org/kernos/496

DOI : $10.4000 /$ kernos.496

ISSN : 2034-787

\section{Éditeur}

Centre international d'étude de la religion grecque antique

Édition imprimée

Date de publication : 1 janvier 2006

Pagination : 485-488

ISSN : 0776-3824

Référence électronique

André Motte, "Christoph Riedweg, Pythagoras. His Life, Teaching, and Influence », Kernos [En ligne], 19। 2006, mis en ligne le 22 mars 2011, consulté le 24 août 2022. URL : http://journals.openedition.org/ kernos/496 ; DOI : https://doi.org/10.4000/kernos.496 
qui suit, démontre, selon V. Liapis, l'application de ces conceptions de Sophocle dans la pratique poétique.

Tout en acceptant que l'attitude que l'A. attribue à Sophocle est plausible, nous pensons qu'il reste un peu trop influencé par des conceptions épistémologiques propres aux Néoplatoniciens et aux Pères de l'Église chrétienne (dont il fait état luimême: p. 55 et 63-64). Son analyse des poètes avant Sophocle et des philosophes avant Socrate, qui ne manque pas de finesse à plusieurs égards, présente également, à notre avis, des anachronismes ou des interprétations qui ne sont pas toujours soutenus par les textes. Il reste que l'ensemble de l'ouvrage constitue une contribution intéressante à l'exploration de ce sujet délicat et épineux : la possibilité pour l'homme de connaître la vérité en général et la vérité sur la divinité en particulier.

Aikaterini Lefka

(Université de Liège - Université de Luxembourg - Towson University)

Christoph Riedweg, Pythagoras. His Life, Teaching, and Influence. Translated from the German by Steven Rendall, Ithaca/London, Cornell University Press, 2005. 1 vol. $16 \times 23,5 \mathrm{~cm}, x i+184$ p. ISBN : 0-8014-4240-0.

Si la figure fascinante de Pythagore a toujours intrigué les historiens de l'antiquité, la documentation ancienne relative à sa personnalité et à son œuvre est tellement prolifique et, pour une grande part, si sujette à caution qu'on n'est pas surpris par le nombre réduit de spécialistes qui ont pris jusqu'ici le risque de lui consacrer toute une monographie et ont tenté d'évaluer l'influence persistante qu'il a exercée. C.R. relève ce défi avec une tranquille assurance, mâtinée de prudence. Sa démarche est sobre et méthodique, s'appuyant sur de nombreux textes cités en traduction; son information est très à jour, servie par une abondante bibliographie; son argumentation est ferme, tout en évitant la polémique. L'édition allemande de l'ouvrage, dédié à W. Burkert, date de 2002. Auparavant déjà, l'A. avait publié plusieurs études sur le penseur de Samos et sur certains de ses disciples, dans le Neue Pauly notamment.

La dimension du volume suggère d'emblée qu'on n'a pas affaire ici à une somme complète du pythagorisme, qu'il s'agisse de la vie du fondateur, de son enseignement et de sa survivance. Du moins offre-t-il sur ces différents sujets un riche aperçu sous la forme d'une mise au point qui n'esquive pas les questions difficiles. Par quantité de traits, on le sait, Pythagore et le pythagorisme relèvent de l'histoire religieuse. C'est cet aspect que retiendra plus particulièrement le présent compte rendu. Le premier des quatre chapitres qui composent l'ouvrage passe rapidement en vue, sans chercher pour l'heure à faire un tri définitif entre " Fiction and Truth », les nombreuses histoires anciennes qui dépeignent notre énigmatique personnage aux facettes multiples. Sont entre autres évoqués la séduction qui émanait de sa personne et aussi de son accoutrement insolite (robe blanche, couronne d'or, etc.), son pouvoir miraculeux sur les animaux sauvages et sur la nature, ses dons de bilocation, de divination et de maitrise d'autres phénomènes parapsychologiques (en particulier la capacité qu'il avait de se souvenir de ses existences antérieures). Il n'est guère étonnant qu'on en soit venu à lui assigner une origine divine, et plus précisément apollinienne. C'est ainsi que Jamblique fait état d'une sorte d'annonciation dont sa mère Pythaïs aurait bénéficié de la part de la pythie de Delphes. Pythaïs s'appelait au préalable Parthenis, «Vierge »: on devine le modèle chrétien qui a pu inspirer cette anecdote! Le nom de Pythagore donné au fils en dit long aussi sur les rapports privilégiés auxquels celui-ci était promis avec l'Apollon Pythios, patron de cet oracle fameux dont les recommandations, est-il dit ailleurs, allaient s'inspirer la doctrine éthique de notre philosophe. Au demeurant, 
on trouve aussi à Crotone, où il débarqua au début de son exil, un sanctuaire dédié à ce même dieu ainsi nommé. On n'est pas davantage surpris d'apprendre que, dans cette même cité de Grande Grèce, il avait fait offrande à Héra Lacinia; c'est que son île natale de Samos était elle-même renommée pour abriter un des plus anciens sanctuaires voués à cette déesse et que la voie sacrée qui y conduisait partait précisément du bourg où il aurait vu le jour (et qui se nomme aujourd'hui Pythagorion!). S'il est une vertu du maître que ses disciples ont mis constamment en exergue, c'est bien sa piété. Le genre de vie qu'il menait, nous dit-on, celle qu'il prêchait aussi à ses adeptes, était aimantée par la volonté de plaire à la divinité; pour pouvoir répondre adéquatement à cette question, il avait même institué une sorte de méthodologie. On sait, d'autre part, les interdictions alimentaires et autres tabous en usage dans la secte, l'importance accordée aussi aux purifications; aux yeux du maître, la pureté valait aux enfants d'être chéris des dieux. Il avait une prédilection pour les lieux saints, en particulier les bosquets sacrés, et recommandait à ses disciples de les fréquenter. Sa dévotion spéciale pour les Muses est bien connue et est à mettre en relation, non seulement avec son intérêt pour la musique et les observations savantes qu'il en tira, mais aussi avec ses conceptions politiques valorisant l'idée de concorde, avec son idéal éthique de purification et avec ses spéculations cosmologiques qui touchent à l'harmonie des sphères et aux sons qu'elles font entendre dans le ciel. La mort de Pythagore, sans doute interprétée comme une sorte d'apothéose, fut mise elle-même en relation avec un sanctuaire des Muses à Métaponte.

Les nombreux voyages qu'on lui impute avant son installation en Grande Grèce lui auraient permis d'être initié par les prêtres égyptiens, à la géométrie notamment, d'être informé par les Perses de la religion de Zoroastre, de recevoir des Chaldéens des connaissances astronomiques, des Phéniciens un savoir arithmétique et des Juifs l'art d'interpréter les songes. Il aurait aussi fréquenté les Celtes et les Ibères, sans compter qu'en Grèce même, il aurait accompagné Épiménide dans un pèlerinage à l'antre crétois de Zeus, sur le mont Ida, où il aurait été de surcroît purifié par les Dactyles. On lui attribue également des contacts avec Éleusis et, en Thrace, une initiation aux mystères d'Orphée par les soins du mystagogue Agloaphamos. Si l'on ajoute que, durant sa jeunesse, Pythagore avait été, dit-on, l'auditeur des Milésiens Thalès et Anaximandre, qu'il aurait en outre côtoyé Phérécyde de Syros dont il partageait certaines idées au sujet de l'âme, on voit qu'à diverses époques disciples et biographes anciens se sont pour ainsi dire donné le mot pour faire de ce personnage surhumain le dépositaire d'une sagesse polyvalente à forte connotation religieuse et censée s'être nourrie à toutes les bonnes sources reconnues de son temps. Ils ont fait pareillement de lui l'inventeur d'un nouveau genre de vie, garant d'un salut d'un type nouveau, et le guru d'une secte où l'on cultivait le secret et dont la brutale dispersion, survenue après un succès flamboyant rencontré en Grande Grèce, n'empêcha pas que soient relayés dans la suite avec vénération l'enseignement du maître. Mais nombreux sont, parmi les auteurs anciens, ceux qui attribuent conjointement au mage de Samos un apport considérable dans les domaines de la philosophie et des sciences, spécialement des mathématiques. Il faut noter aussi qu'il n'a pas eu que des admirateurs tentés par le genre hagiographique, mais qu'il a été très tôt un signe de contradiction : on était pour ou contre lui. De son vivant, sans même parler des graves tribulations que sa secte suscita en Grande Grèce, il a attiré des propos ironiques et des sarcasmes, de la part notamment de confrères philosophes.

Que retenir de tout ce ramassis de traditions et de légendes pour camper le portrait d'un Pythagore un tant soit peu authentique? Ne désespérant pas d'atteindre sur cette question très controversée quelque résultat probant, C.R., dans un deuxième chapitre 
joliment intitulé "In Search of the Historical Pythagoras », remet méthodiquement l'ouvrage sur le métier, sans pouvoir éviter quelques redites. Après avoir rapidement évoqué le milieu culturel, marqué à l'époque par d'importantes avancées, tout particulièrement dans cette Ionie largement ouverte sur l'Orient et aussi l'Occident, il commence par examiner les témoignages les plus anciens qui concernent notre penseur, - Xénophane, Héraclite, Ion de Chios, Empédocle, Hérodote, Démocrite, puis les témoins du début du $\mathrm{IV}^{\mathrm{e}} \mathrm{s}$., - dans l'espoir de pouvoir se forger ainsi des critères qui permettront de filtrer les témoignages plus récents. Voilà qui est sans doute de bonne méthode, mais qui ne semble pas fournir en l'occurrence des points de repère aussi assurés qu'on eût pu le souhaiter. Pour le dire d'emblée, car c'est là peut-être un des principaux enjeux du travail, la conclusion à laquelle aboutit le chapitre est que, si Pythagore fut assurément le guru d'une secte qu'il a lui-même fondée et un personnage éminemment charismatique, il fut plus que cela. Autrement dit, prenant quelque distance à l'égard de ceux qui, comme Burkert, voyaient l'intéressé sous les traits d'un chaman et répugnaient à le créditer d'un quelconque profil scientifique, Riedweg considère pour sa part que dimension mystique et dimension scientifique ont fort bien pu chez lui coexister et se conforter, selon un processus analogue à ce que les anthropologues ont mis en lumière à propos de la «pensée sauvage » et comme en témoigne aussi l'histoire des sciences et de la philosophie (qu'il me soit permis de conseiller à ce propos la lecture de l'ouvrage de Bertrand Russell, Histoire de mes idées philosophiques, Gallimard, 1961, dont un chapitre intitulé «La renonciation à Pythagore » évoque l'engouement vraiment mystique qui fut un moment le sien pour les mathématiques). C.R. parvient à cette conclusion au terme d'un examen systématique des principaux aspects du pythagorisme, parcours très ordonné qui fait largement confiance aux témoignages d'Aristote et de ses disciples et qui lui permet, avec nuances, de faire la part de ce qui peut être attribué au fondateur. Sont notamment examinés plusieurs points de rencontre avec l'orphisme. Au total, sans que soit du tout abolie l'ambiguiité du personnage, c'est une pensée assez consistante qui lui est reconnue, caractérisée par une conception mathématique de l'univers alliée à une doctrine religieuse de salut qui appelle un genre de vie ascétique. C.R. n'exclut pas l'idée que Pythagore ait pu se donner à lui-même le titre nouveau de philosophe, la vie philosophique qu'il préconise accordant une primauté à la theoria, l'activité de contemplation.

Des deux chapitres restants, le premier est consacré à la société secrète pythagoricienne. L'A. montre tout d'abord que cette société répond bien à la qualification de secte, au sens que donne à ce mot la sociologie des religions. Il examine ensuite ce qu'a été sa destinée aux $\mathrm{v}^{\mathrm{e}}$ et $\mathrm{IV}^{\mathrm{e}}$ s., - les rébellions anti-pythagoriciennes mais aussi les tensions internes entre akousmatikoi et mathèmatikoi, - puis il passe en revue les membres principaux, en s'attardant davantage aux deux figures de proue qu'ont été Philolaos et Archytas. Le dernier chapitre esquisse une histoire de l'influence que Pythagore a exercée sur la pensée occidentale, depuis les pré-platoniciens jusqu’à l'«Harmonical Pythagoreanism », courant de pensée dont le musicien et historien de l'art Hans Keyser fut encore, au $\mathrm{xx}^{\mathrm{e}} \mathrm{s}$. un représentant attitré et qui est sans doute redevable à Pythagore de certaines conceptions musicales. Un problème majeur soulevé dans cette section, et qui a fait déjà couler beaucoup d'encre, concerne l'influence que ce dernier a pu avoir sur Platon. Précédemment déjà, C.R. avait observé que le fait d'avoir pu compter sur un penseur de la taille et de l'audience du fondateur de l'Académie pour relayer ses conceptions fut pour le pythagorisme une aubaine peu banale. Il avait montré en outre que l'héritage pythagoricien qui survit à l'époque hellénistique et qui reprend vigueur au début de notre ère ainsi qu'à l'âge du néopla- 
tonisme, est un pythagorisme très platonisant. C'est à tort, par exemple, que certains néoplatoniciens attribuent à Pythagore la conception platonicienne de nombres séparés du monde sensible. On ne peut qu'acquiescer à ces justes observations, d'autant que C.R. n'entend nullement minimiser par là l'empreinte que le pythagorisme a laissée dans la philosophie de Platon. Dans le bilan un peu rapide que dresse à ce sujet le dernier chapitre, il eût été opportun cependant de rappeler, à côté d'une série de rapprochements précis et pertinents sur lesquels on n'attire pas souvent l'attention, que c'est aussi dans le sillage de Pythagore que Platon adhère à une anthropologie dualiste âme-corps, accueille la conception de l'immortalité de l'âme et de ses réincarnations, développe une eschatologie céleste et tend à présenter lui aussi la philosophie comme une doctrine de salut qui privilégie l'idéal de contemplation et est fortement imprégnée de religiosité.

En frontispice du premier chapitre figure une carte géographique montrant l'aire de diffusion du pythagorisme dans l'antiquité, tandis qu'à la fin du volume, précédant les notes (qui se réduisent le plus souvent à des références abrégées aux auteurs anciens et modernes) et la liste des abréviations, un tableau chronologique présente, dans son environnement culturel, le développement du pythagorisme depuis sa fondation jusqu'à l'époque moderne. Suivent une bibliographie de près de 300 titres ainsi qu'un index général très copieux. S'ajoutant à l'apport substantiel des chapitres, ces compléments utiles font que cet ouvrage introductif sera très apprécié par tous ceux qui s'intéressent à Pythagore et à l'impact majeur que, par une série de relais successifs, il a eu sur le développement de la culture occidentale.

André Motte (Université de Liège)

Renée Koch, Comment peut-on être dieu? La secte d'Épicure, Paris, Belin, 2005. 1 vol. $14 \times 21$ cm, 300 p. (L'Antiquité au présent). ISBN : 2-7011-4024-2.

Voici un ouvrage qui ne court guère le risque de passer inaperçu. Ce qu'on tient aujourd'hui pour «scientifiquement correct", à tout le moins dans notre ère culturelle francophone, ne prédispose guère, en effet, à marier le philosophique et le religieux. Or le dessein que poursuit l'A., et que le titre choisi met d'emblée en relief, est de faire voir au cour même de l'épicurisme antique, et tout au long des quelque cinq siècles où il a fait fortune, une véritable religion philosophique. Ce n'est pas la première fois que l'on rame ainsi à contre-courant, mais, déployant une remarquable érudition, R.K. explore des pistes jusqu'ici peu fréquentées et propose des arguments nouveaux.

Dans une introduction très dense, elle dit son intention de rattacher l'épicurisme à l'histoire des religions et dénonce le préjugé moderne qui oppose, dans la foulée des Lumières, « le religieux au rationnel, comme l'archaïque au moderne et comme l'affect à l'intellect » (p. 7). C'est bien à tort que, cédant à cette opposition, certains se plaisent à saluer dans l'atomisme matérialiste des Épicuriens, héritage de Leucippe et Démocrite, l'apparition d'un logos universel «écartant les ronces de la religion », «un fleuron de la rationalité »(p. 8) demeuré à l'abri des pratiques magiques et des croyances superstitieuses comme des poussées spiritualistes que l'on doit à Platon, à Aristote et aux Stö̈ciens. À l'inverse de Cornford qui considère que les dieux des atomistes "se sont évanouis dans la danse des particules matérielles », l'A. estime qu'avec Épicure «ils se sont au contraire manifestés plus clairement que jamais » (p. 14), la secte s'étant donné les moyens de les garder constamment en vue dans la fin qu'elle poursuit. L'enquête historique et anthropologique qu'elle a menée révèle de façon continue, chez les Épicuriens, un comportement pieux qui, loin de signifier une 